\title{
Estimates of Genetic Variability of Mycobacterium tuberculosis Complex and Its Association with Drug Resistance in Cameroon
}

\author{
Larissa Kamgue Sidze ${ }^{1,2}$, Emmanuel Mouafo Tekwu ${ }^{1,2}$, Christopher Kuaban ${ }^{3}$, \\ Jean-Paul Assam Assam ${ }^{1}$, Jean-Claude Tedom ${ }^{1}$, Stefan Niemann ${ }^{4}$, Matthias Frank ${ }^{2}$, \\ Véronique N. Penlap Beng ${ }^{*}$ \\ ${ }^{1}$ Laboratory for Tuberculosis Research, Biotechnology Centre of Nkolbisson (BTC), University of Yaoundé I, Yaoundé, Cameroon; \\ ${ }^{2}$ Institute for Tropical Medicine, University of Tübingen, Tübingen, Germany; ${ }^{3}$ Pneumology Unit, Jamot Hospital, Yaoundé, Cam- \\ eroon; ${ }^{4}$ Research Centre Borstel, Molecular Mycobacteriology, Borstel, Germany. \\ Email: *v.penlap@yahoo.fr
}

Received November $5^{\text {th }}, 2012$; revised December $8^{\text {th }}, 2012$; accepted January $1^{\text {st }}, 2013$

\begin{abstract}
The present study investigates the genetic diversity among Mycobacterium tuberculosis complex circulating in the Centre region of Cameroon and analyzes the relationship between genotypes and drug resistance patterns. Spoligotyping was performed by PCR-amplification followed by the reverse hybridization of 298 cultured specimens. Spoligotypes patterns were identified by comparison to reference strains in SPolDB4 database via the MIRU VNTR plus web application. About $97.65 \%$ of all tuberculosis (TB) cases were attributed to M. tuberculosis. A total of 65 different profiles were identified. Of these, 40 were represented as Shared Types (ST) while the others were orphans. LAM10_CAM and Haarlem families were the most prevalent genetic families with $51.01 \%$ and $14.09 \%$ respectively. ST 61 , a member of the LAM10_ CAM family formed the largest cluster with 128 (42.95\%) isolates. No association was found between genotypes with regard to drug resistance and HIV sero-status. However, there was a significant association between genotypes and age groups. Patients belonging to $15-24$ and $35-44$ age groups were more likely infected by LAM10_CAM strains compared to others. The population structure of Mycobacterium tuberculosis complex strains from the Centre region was found to be diverse and the spoligotype 61 of the LAM10_CAM family was highly predominant. Isolates of the LAM10_CAM seem to be not associated with drug resistance.
\end{abstract}

Keywords: M. tuberculosis; Spoligotyping; LAM10_CAM

\section{Introduction}

Tuberculosis (TB) is a cause of great mortality and suffering, especially in poor and less-developed countries. Its association with the HIV/AIDS pandemic forms a lethal combination. In addition, multidrug resistant (MDR) TB and extensively drug resistant (XDR) TB severely complicate the management and control of the disease worldwide [1]. More recently, the discovery of totally drug resistant (TDR) TB, a deadly form of the disease highlighted a crisis of mismanagement of the disease [2]. Elimination of TB by 2050 is a long-term goal of the World Health Organization (WHO) and their strategy is heavily based on the improvements in the current diagnostics, treatment and vaccination, as well as on the development of new strategies to control and fight the epidemic [3]. Any strategy for combating the epidemic should be based on a thorough appreciation of the pro-

${ }^{*}$ Corresponding author. blem. Interventions driven by a poor understanding of the pathogen in a specific geographical context will necessarily entail a high risk of failure [4].

Our understanding of the transmission of tuberculosis (TB) has been greatly enhanced since the introduction of DNA fingerprinting techniques for Mycobacterium tuberculosis [5]. Spoligotyping is a very practical and reproducible PCR-based method, which assays the presence or the absence of a set of target sequences in the direct repeat (DR) locus [6]. The resulting genotype has a simple binary format, which has recently leaded to the construction of large databases, intended to facilitate recognition of the origin of a particular clinical isolate [7]. Another advantage of spoligotyping is that it can be used simultaneously for the detection and typing of the $M$. tuberculosis complex bacteria in one assay.

As in most resource poor countries, TB epidemiology in Cameroon has so far largely consisted of reporting the 
number of cases detected and their demographic data. Little is known about Mycobacterium tuberculosis strains circulating in the Centre region of the country. The objective of the present study was to estimate the genetic variability of Mycobacterium tuberculosis complex strains circulating in the Centre region and to analyze the relationship between genotypes and drug resistance.

\section{Materials and Methods}

\subsection{Mycobacterial Isolates}

This study included 298 Mycobacterium tuberculosis complex isolates. These isolates were selected from a collection of Mycobacterium tuberculosis complex strains isolated from smear positive pulmonary tuberculosis patients admitted in Jamot hospital and Mbalmayo District hospital, whose age was ranged from 15 to 85 (mean age, 33.75 years). Among the selected isolates, 28 (10.64\%) were phenotypically drug resistant and 3 were multidrug resistant. HIV serology was available for 296 (99.32\%), among which 86 (29.05\%) were HIV positive.

\subsection{DNA Extraction}

Mycobacterium tuberculosis complex were scraped from Lowestein-Jensen slopes, collected into Eppendorf tubes containing Tris-EDTA ( $10 \mathrm{mM}, 1 \mathrm{mM}, \mathrm{pH}$ 8) and heated for $30 \mathrm{~min}$ at $90^{\circ} \mathrm{C}$. After centrifugation at $13,000 \mathrm{xg}$, the supernatant was collected into a new tube and kept at $-20^{\circ} \mathrm{C}$ until further use.

\subsection{Spoligotyping}

All isolates were genotyped with a spoligotyping commercial kit (Isogen Bioscience, BV Maarsen, The Netherlands) according to the protocol previously described by Kamerbeek et al. [6]. Briefly, the DR region of the TB genome was amplified using primers DRa,

5'-GGTTTTGGGTCTGACGAC-3' (biotinylated 5' end) and DRb, -CCGA-GAGGGGACGGAAAC-3'.

PCR products were hybridized with a set of 43 spacer oligonucleotides covalently linked to the spoligo-membrane (Isogen Life Sciences, The Netherlands) according to the manufacturer's instructions. The hybridized PCR products were then incubated with a streptavidin-peroxidase conjugate and the membrane exposed to chemiluminescence (Amersham ECL Direct ${ }^{\mathrm{TM}}$ nucleic acid labeling and detection system, GE Healthcare Limited, UK). The X-ray film was developed using standard photochemical procedures after 20 minutes exposure. DNA extracts of M. tuberculosis $\mathrm{H} 37 \mathrm{Rv}$ and M. bovis BCG were used as controls.

\subsection{Data Analysis}

Spoligotype patterns in a binary format were entered in an Excel sheet, and compared with the spoligotype database SpolDB4 using MIRU VNTR plus [8]. The Hunter Gaston Discriminatory Index (HGDI) was used to calculate the discriminatory power of spoligotyping method [9]. The Chi square or Exact Fisher test when necessary were employed to evaluate difference in serology, age group and drug resistance between LAM10_CAM and non LAM10_CAM strains. Values of $p$ of less than 0.05 were considered significant.

\section{Results}

Of the 298 isolates analyzed, 291 (97.65\%) were classified as Mycobacterium tuberculosis and $6(2.03 \%)$ as Mycobacterium africanum species. The remaining one isolate was identified as Mycobacterium bovis.

\subsection{Distribution of Different Genetic Families}

Among the 298 typed isolates, a total of 65 different profiles clustered into 17 genetic families were identified. Of these, $152(51.01 \%)$ isolates belong to the LAM10_CAM family while 121 (42.7\%) were non LAM10_CAM strains. Strains classified into non LAM10_CAM family included strains from the Haarlem family (14.09\%), T family (12.75\%) and others (Table 1).

Table 1. Distribution of different genetic families identified in a collection of 298 Mycobacterium tuberculosis complex isolates.

\begin{tabular}{ccc}
\hline Genetic families & No. isolates & Frequency $(\%)$ \\
\hline LAM10_CAM & 152 & 51.01 \\
H3 & 42 & 14.09 \\
T2 & 22 & 7.38 \\
U & 14 & 4.70 \\
T1 & 12 & 4.03 \\
LAM1 & 6 & 2.01 \\
U (likely H) & 6 & 2.01 \\
AFRI_2 & 3 & 1.01 \\
H1 & 3 & 1.01 \\
LAM9 & 3 & 1.01 \\
U (likely H3) & 2 & 0.67 \\
AFRI & 1 & 0.34 \\
AFRI_1 & 1 & 0.34 \\
AFRI_3 & 1 & 0.34 \\
CAS1_DELHI & 1 & 0.34 \\
T1 (T4-CE1 ancestor0) & 1 & 0.34 \\
T2-T3 & 1 & 0.34 \\
T5 & 1 & 0.34 \\
T5_MAD2 & 1 & 0.34 \\
Non identified & 25 & 8.39 \\
Total & 298 & 100.00 \\
\hline & &
\end{tabular}




\subsection{Predominant Spoligotypes}

Of the 65 spoligotypes identified, 40 were represented as Shared Types (ST) according to SpolDB4 while the others were reported for the first time. Among these Shared Types, ST 61 member of the LAM10_CAM and ST 50 member of the Haarlem family respectively represented $42.95 \%$ and $11.41 \%$ (Table 2).

The HGDI value for spoligotyping was low (79.72\%), especially for the strains of the LAM10_CAM family
(96.71\%). No correlation was found between the identified genotypes with regard to drug resistance, and HIV sero-status (Table 3). However, a statistical association was found between the LAM10_CAM isolates and age groups. Patients belonging to $1 \overline{5}-24$ and $35-44$ age groups were more likely infected by LAM10_CAM strains compared to others. In the LAM10_CAM family, the distribution of different Share Types ST403, ST61, ST838, ST850 and ST852 was not associated with HIV sero-status.

Table 2. Distribution of Share Types (ST) identified in a collection of 298 Mycobacterium tuberculosis complex isolates.

\begin{tabular}{|c|c|c|c|c|}
\hline Genotypes & Share-Types (ST) & Spoligo-patterns & No. isolates & Frequency (\%) \\
\hline AFRI & 332 & 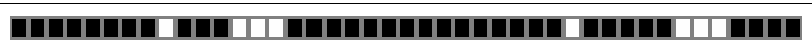 & 1 & 0.34 \\
\hline AFRI_1 & 715 & ต & 1 & 0.34 \\
\hline \multirow[t]{2}{*}{ AFRI_2 } & 101 & mum & 1 & 0.34 \\
\hline & 331 & Gmum & 2 & 0.67 \\
\hline \multirow[t]{2}{*}{ AFRI_3 } & 856 & mum & 1 & 0.34 \\
\hline & 1223 & 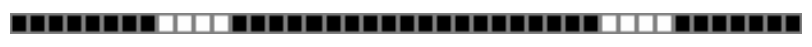 & 2 & 0.67 \\
\hline \multirow{3}{*}{$\mathrm{T} 1$} & 1324 & 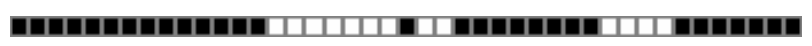 & 2 & 0.67 \\
\hline & 144 & mmmm & 1 & 0.34 \\
\hline & 53 & 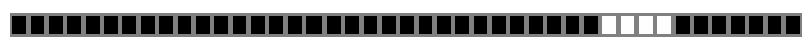 & 7 & 2.35 \\
\hline \multirow[t]{3}{*}{ T1 (T4-CE1 ancestor) } & 65 & 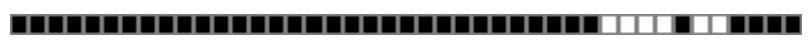 & 1 & 0.34 \\
\hline & 1056 & 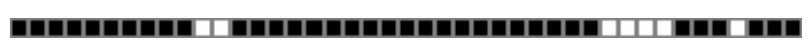 & 2 & 0.67 \\
\hline & 125 & 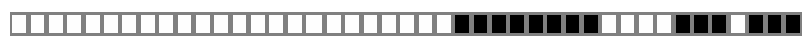 & 1 & 0.34 \\
\hline \multirow{4}{*}{$\mathrm{T} 2$} & 317 & 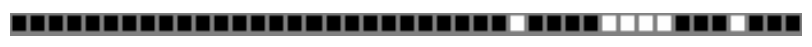 & 2 & 0.67 \\
\hline & 52 & $\mathbf{m}=10$ & 8 & 2.68 \\
\hline & 848 & 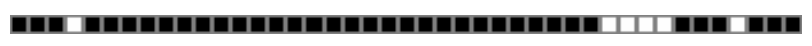 & 1 & 0.34 \\
\hline & 853 & 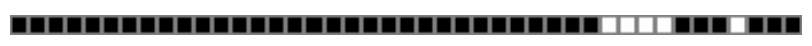 & 8 & 2.68 \\
\hline $\mathrm{T} 2-\mathrm{T} 3$ & 73 & 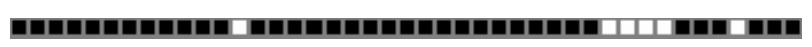 & 1 & 0.34 \\
\hline $\mathrm{T} 5$ & 44 & 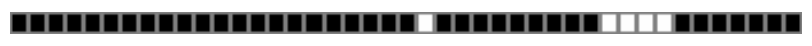 & 1 & 0.34 \\
\hline T5_MAD2 & 1227 & 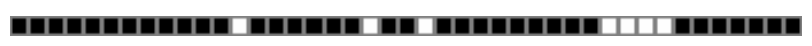 & 1 & 0.34 \\
\hline LAM1 & 20 & 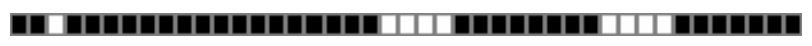 & 6 & 2.01 \\
\hline \multirow[t]{3}{*}{ LAM9 } & 42 & 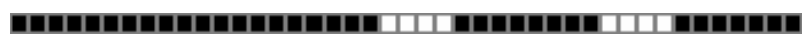 & 3 & 1.01 \\
\hline & 403 & 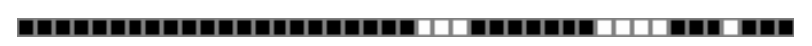 & 3 & 1.01 \\
\hline & 61 & 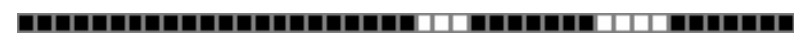 & 128 & 42.95 \\
\hline \multirow[t]{3}{*}{ LAM10_CAM } & 838 & 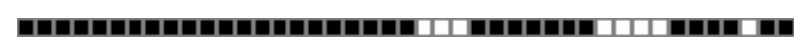 & 14 & 4.70 \\
\hline & 850 & 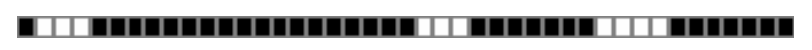 & 5 & 1.68 \\
\hline & 852 & 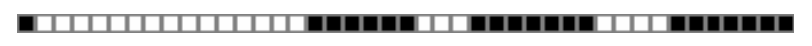 & 2 & 0.67 \\
\hline \multirow[t]{4}{*}{ H1 } & 47 & [um & 3 & 1.01 \\
\hline & 316 & 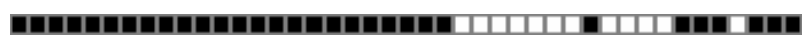 & 4 & 1.34 \\
\hline & 49 & Gum & 1 & 0.34 \\
\hline & 50 & 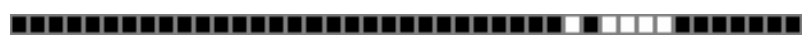 & 34 & 11.41 \\
\hline \multirow{3}{*}{$\mathrm{H} 3$} & 75 & Bum & 1 & 0.34 \\
\hline & 840 & 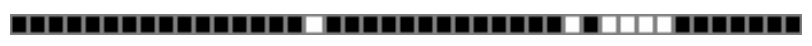 & 1 & 0.34 \\
\hline & 99 & प Пل & 1 & 0.34 \\
\hline \multirow[t]{2}{*}{ CAS1_DELHI } & & m & 1 & 0.34 \\
\hline & 124 & 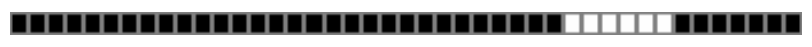 & 1 & 0.34 \\
\hline \multirow{3}{*}{$\mathrm{U}$} & 450 & 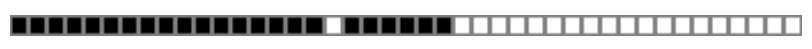 & 11 & 11.41 \\
\hline & 786 & 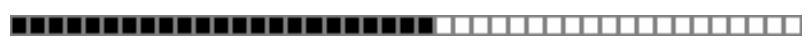 & 1 & 0.34 \\
\hline & 839 & 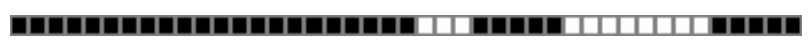 & 1 & 0.34 \\
\hline \multirow{2}{*}{ U (likely H) } & 46 & 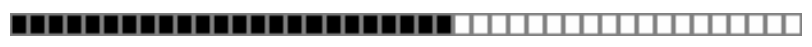 & 6 & 2.01 \\
\hline & 237 & 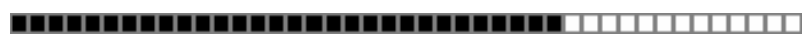 & 2 & 0.67 \\
\hline
\end{tabular}


Table 3. Distribution of LAM10_CAM and non LAM10_CAM genotype according to drug resistance, age group and HIV sero-status.

\begin{tabular}{|c|c|c|c|c|c|c|}
\hline & $\begin{array}{c}\text { Total } \\
\mathrm{N}=\mathbf{2 9 8} \\
\end{array}$ & $\begin{array}{c}\text { LAM10_CAM } \\
\mathrm{N}=152\end{array}$ & $\%$ & $\begin{array}{c}\text { Non LAM10_CAM } \\
\mathbf{N}=146\end{array}$ & $\%$ & p-value \\
\hline \multicolumn{7}{|l|}{ DST results } \\
\hline Resistant & 28 & 17 & 60.71 & 11 & 39.29 & \multirow{2}{*}{0.28} \\
\hline Susceptible & 270 & 135 & 50.00 & 135 & 50.00 & \\
\hline \multicolumn{7}{|l|}{ Age groups } \\
\hline $15-24$ & 72 & 46 & 63.87 & 26 & 36.13 & $0.01^{*}$ \\
\hline $25-34$ & 103 & 51 & 49.51 & 52 & 50.49 & 0.70 \\
\hline $35-44$ & 72 & 28 & 38.88 & 44 & 61.12 & $0.02^{*}$ \\
\hline $45-54$ & 28 & 18 & 64.28 & 10 & 35.72 & 0.14 \\
\hline $55-64$ & 23 & 9 & 39.13 & 14 & 60.87 & 0.23 \\
\hline \multicolumn{7}{|c|}{ HIV sero-status } \\
\hline Positive & 86 & 50 & 58.14 & 36 & 41.86 & \multirow{2}{*}{0.07} \\
\hline Negative & 274 & 98 & 35.76 & 111 & 64.23 & \\
\hline
\end{tabular}

*Statistically significant.

\section{Discussion}

It has been reported in some instances that, spoligotyping can distinguish among members of the $M$. tuberculosis complex based on the species-specific presence/absence of spacers [10]. In our study, 3 different species were identified among $298 \mathrm{M}$. tuberculosis complex isolates. With more than $90 \%$ cases, $M$. tuberculosis was far the most prevalent species. A similar observation was reported among the M. tuberculosis complex isolates collected from the West region of Cameroon [11].

The comparison of spoligotypes found in this study with the International Spoligotyping Database SPolDB4, showed that the most prevalent spoligotype was ST 61 followed by ST 50, which belong respectively to the LAM10_CAM family and Haarlem family. A similar predominance of the LAM10_CAM family was previously described among Mycobacterium tuberculosis isolates from Burkina faso [12] and Benin [13]. Although this genotype was described to be prevalent in some countries of the West African coast [11], a study conducted in Sierra Leone revealed only $4 \%$ of strains belonging to the LAM10_CAM [14]. The factors that might contribute to the adaptability of $M$. tuberculosis strains or lineages to a particular population or zone are poorly understood. As hypothesized for the Tunisian family [15], mass BCG vaccination strictly applied for decades might have profoundly shaped the population structure of $M$. tuberculosis by concurrently favoring the selection and accommodation of particular genotypes, as the LAM10 CAM family in our setting.
W-Beijing family strains were not identified in our isolates. It has been reported that this genotype is very rare in some West African coast countries [12]. A proportion of $10.3 \%$ was reported among M. tuberculosis from Cotonou (Benin) [13]. As expected, the HGDI value for spoligotyping was low $(79.72 \%)$. To increase the discriminatory power and for a better understanding of the molecular diversity of the studied population, a more discriminatory technique such as MIRU-VNTR typing is recommended [18].

As it was reported in some instances that, genotypes like W-Beijing family are associated with drug resistance [17], we analyzed the relationship between genotypes circulating in our setting and drug resistance. However, we did not find any statistical association between genotypes and drug resistance $(\mathrm{p}=0.47)$ even HIV serostatus $(p=0.07)$ in our study, but only with age groups $(p=$ 0.01 ). The distribution of the LAM10_CAM strains according to age groups followed the trend of HIV infection in our study population. It has been reported that strains well adapted to a specific population like the LAM10 CAM family are more likely to transmit compared to others [18]. Since HIV infection, a known risk factor for tuberculosis was associated with age groups; this could explain the association of LAM10_CAM strains with age groups. Five different Share Types ST403, ST61, ST838, ST850 and ST852 were identified in the LAM10 CAM family. The distribution of these genotypes did not correlate with HIV sero-status. This observation was previously reported in isolates from the West region of the country [11]. 
The population structure of Mycobacterium tuberculosis complex strains from the Centre region was diverse and included 65 different genotypes. The majority of strains belonged to the LAM10_CAM which can be subdivided in 5 spoligotypes. The consequence of this diversity for the TB epidemic are not yet clear and need to be addressed in further studies.

\section{Acknowledgements}

This study was financially supported by EDCTP grant through the CANTAM-TB "Central Africa Network for Tuberculosis, AIDS/HIV and Malaria" project. Larissa Kamgue Sidze and Emmanuel Mouafo Tekwu were research fellow students at the Institute for Tropical Medicine in Tübingen (Germany). We thank Mrs. Augusta Tsasse (Centre Pasteur of Cameroon) and Tanja Ubben (Research Centre Borstel, Germany) for their technical assistance.

\section{REFERENCES}

[1] C. Dye, "Global Epidezmiology of Tuberculosis," Lancet, Vol. 367, No. 9514, 2006, pp. 938-940. doi:10.1016/S0140-6736(06)68384-0

[2] K. Rowland, "Totally Drug-Resistant TB Emerges in India," Nature News and Comment, 13 January 2012.

[3] World Health Organization, "Global Tuberculosis Control: WHO Report 2011," Geneva, 2011.

[4] G. D. Van der Spuy, R. M. Warren and P. D. Van Helden, "The Role of Molecular Epidemiology in Low-Income, High-Burden Countries," International Journal of Tubercle and Lung Diseases, Vol. 13, No. 4, 2009, pp. 419420 .

[5] D. Van Soolingen, P. W. Hermans, P. E. De Haas, D. R. Soll and J. D. Van Embden, "Occurrence and Stability of Insertion Sequences in Mycobacterium tuberculosis Complex Strains: Evaluation of an Insertion Sequence-Dependent DNA Polymorphism as a Tool in the Epidemiology of Tuberculosis," Journal of Clinical Microbiology, Vol. 29, No. 11, 1991, pp. 2578-2586.

[6] J. Kamerbeek, L. Schouls, A. Kolk, M. Van Agterveld, D. Van Soolingen, S. Kuijper, A. Bunschoten, H. Molhuizen, R. Shaw, M. Goyal, et al., "Simultaneous Detection and Strain Differentiation of Mycobacterium tuberculosis for Diagnosis and Epidemiology," Journal of Clinical Microbiology, Vol. 35, No. 4, 1997, pp. 907-914. doi:10.3201/eid0811.020125

[7] I. Filliol, J. R. Driscoll, D. Van Soolingen, B. N. Kreiswirth, K. Kremer, G. Valetudie, D. D. Anh, R. Barlow, D. Banerjee, P. J. Bifani, et al., "Global Distribution of Mycobacterium tuberculosis Spoligotypes," Emerging Infectious Diseases, Vol. 8, No. 11, 2002, pp. 1347-1349.

[8] T. Weniger, J. Krawczyk, P. Supply, S. Niemann and D.
Harmsen, "MIRU-VNTR plus: A Web Tool for Polyphasic Genotyping of Mycobacterium tuberculosis Complex Bacteria," Nucleic Acids Research, Vol. 38, 2010, pp. W326-W331.

[9] P. R. Hunter and M. A. Gaston, "Numerical Index of the Discriminatory Ability of Typing Sysems: An Application of Simpson's Index of Diversity," Journal of Clinical Microbiology, Vol. 26, No. 1, 1988, pp. 2465-2466.

[10] B. Mathema, N. E. Kurepina, P. J. Bifani and B. N. Kreiswirth, "Molecular Epidemiology of Tuberculosis: Current Insights," Clinical Microbiology Review, Vol. 19, No. 4, 2006, pp. 658-685. doi:10.1128/CMR.00061-05

[11] S. N. Niobe-Eyangoh, C. Kuaban, P. Sorlin, P. Cunin, J. Thonnon, C. Sola, et al., "Genetic Biodiversity of Mycobacterium tuberculosis Complex Strains from Patients with Pulmonary Tuberculosis in Cameroon," Journal of Clinical Microbiology, Vol. 41, No. 6, 2003, pp. 25472553. doi:10.1128/JCM.41.6.2547-2553.2003

[12] S. Godreuil, G. Torrea, D. Terru, F. Chevenet, S. Diagbouga, P. Supply, et al., "First Molecular Epidemiology Study of Mycobacterium tuberculosis in Burkina Faso," Journal of Clinical Microbiology, Vol. 45, No. 3, 2007, pp. 921-927. doi:10.1128/JCM.01918-06

[13] D. Affolabi, G. Anyo, F. Faihun, N. Sanoussi, I. C. Shamputa, L. Rigouts, et al., "First Molecular Epidemiological Study of Tuberculosis in Benin," International Journal of Tubercle and Lung Diseases, Vol. 13, No. 13, 2009, pp. 317-322.

[14] S. Homolka, E. Post, B. Oberhauser, A. G. George, L. Westman, F. Dafae, S. Rusch-Gerdes and S. Niemann, "High Genetic Diversity among Mycobacterium tuberculosis Complex Strains from Sierra Leone," BMC Microbiology, Vol. 8, No. 103, 2008, pp. 489-495.

[15] A. Namouchi, A. Karboul, B. Mhenni, N. Khabouchi, R. Haltiti, R. Ben Hassine, et al., "Genetic Profiling of $M y$ cobacterium tuberculosis in Tunisia: Predominance and Evidence for the Establishment of a Few Genotypes," Journal of Medical Microbiology, Vol. 57, No. 7, 2008, pp. 864-872. doi:10.1099/jmm.0.47483-0

[16] P. Supply, E. Mazars, S. Lesjean, V. Vincent, B. Gicquel and C. Locht, "Variable Human Minisatellite-Like Regions in the Mycobacterium tuberculosis Genome," Molecular Microbiology, Vol. 36, No. 3, 2000, pp. 762-771. doi:10.1046/j.1365-2958.2000.01905.x

[17] W. A. Githui, A. M. Jordaan, E. S. Juma, P. Kinyanjui, F. G. Karimi, J. Kimwomi, et al., "Identification of MDRTB Beijing/W and Other Mycobacterium tuberculosis Genotypes in Nairobi, Kenya," International Journal of Tubercle and Lung Diseases, Vol. 8, No. 3, 2004, pp. 352-360.

[18] S. Gagneux and P. M. Small, "Global Phylogeography of Mycobacterium tuberculosis and Implications for Tuberculosis Product Development," Lancet Infectious Diseases, Vol. 7, No. 5, 2007, pp. 328-337. doi:10.1016/S1473-3099(07)70108-1 